I. MaLinowska (Lublin)

P. PAWLAS (Lublin)

D. SzYnal (Lublin)

\title{
ESTIMATION OF THE PARAMETERS \\ OF GUMBEL AND BURR DISTRIBUTIONS IN TERMS OF $k$ TH RECORD VALUES
}

Abstract. The minimum variance linear unbiased estimators (MVLUE), the best linear invariant estimators (BLIE) and the maximum likelihood estimators (MLE) based on $m$ selected $k$ th record values are presented for the parameters of the Gumbel and Burr distributions.

1. Introduction and preliminaries. We say that a random variable $X$ has the Gumbel distribution with parameters $\mu$ and $\sigma$ if

$$
F(x)=e^{-e^{-(x-\mu) / \sigma}}, \quad x \in \mathbb{R} ; \quad-\infty<\mu<\infty, \sigma>0 .
$$

We say that a random variable $X$ has the Burr distribution with parameters $\mu, \sigma, \beta$ and $\lambda$ if

$$
\begin{aligned}
& F(x)=1-\beta^{\lambda}\left(\beta+\frac{x-\mu}{\sigma}\right)^{-\lambda}, \quad x \geq \mu ; \\
&-\infty<\mu<\infty, \sigma>0, \beta>0, \lambda>0 .
\end{aligned}
$$

From the Burr distribution we get the generalized Pareto distribution $(\lambda=$ $\left.\beta=\alpha^{-1}\right)$ and the Lomax distribution $(\beta=1)$.

In [1] and [2] various estimators of the scale parameter $\sigma$ and the location parameter $\mu$ for various classes of distributions (Gumbel distribution, power distribution, Weibull distribution, Rayleigh distribution, logistic distribution, Pareto distribution) based on record values were given. The Bayesian estimators of the Gumbel parameters $\mu$ and $\sigma$ in terms of lower record values

2000 Mathematics Subject Classification: 62F10, 62F15, 62G99.

Key words and phrases: Gumbel model, Burr distribution, minimum variance linear unbiased estimators, Gauss-Markov theorem, best linear invariant estimators, maximum likelihood estimators, $k$ th record values. 
and $k$ th lower record values were furnished in [6] and [9]. Moreover, estimators for location and scale parameters were given in terms of generalized order statistics (cf. [3]-[5]).

We give the maximum likelihood (MLE), best linear invariant (BLIE), and minimum variance unbiased (MVLUE) estimators of the parameters $\mu$ and $\sigma$ for the Gumbel and Burr distributions using the $k$ th lower and upper record values. The use of record values to construct estimators was discussed in [1] and [2]. Some of those results are generalized in this paper.

We recall the concept of $k$ th upper and lower record values (cf. [7], [11]). Let $\left\{X_{n}, n \geq 1\right\}$ be a sequence of independent identically distributed random variables with a cumulative distribution function $F$ and a probability density function $f$. The $j$ th order statistic of a sample $\left(X_{1}, \ldots, X_{n}\right)$ is denoted by $X_{j: n}$. For a fixed $k \geq 1$ we define the sequence $\left\{U_{k}(n), n \geq 1\right\}$ of $k$ th upper record times as follows:

$$
\begin{aligned}
U_{k}(1) & =1, \\
U_{k}(n+1) & =\min \left\{j>U_{k}(n): X_{j: j+k-1}>X_{U_{k}(n): U_{k}(n)+k-1}\right\}, \quad n \geq 1 .
\end{aligned}
$$

The sequence $\left\{Y_{n}^{(k)}, n \geq 1\right\}$ with $Y_{n}^{(k)}=X_{U_{k}(n): U_{k}(n)+k-1}$ is called the sequence of $k$ th upper record values of $\left\{X_{n}, n \geq 1\right\}$. For $k=1$ we have the sequence $\left\{Y_{n}^{(1)}, n \geq 1\right\}$ of upper record values. The probability density function of $\left(Y_{1}^{(k)}, \ldots, Y_{n}^{(k)}\right)$ is given by

$$
\begin{aligned}
& f_{Y_{1}^{(k)}, \ldots, Y_{n}^{(k)}}\left(x_{1}, \ldots, x_{n}\right) \\
& \quad= \begin{cases}k^{n} \prod_{i=1}^{n-1} \frac{f\left(x_{i}\right)}{1-F\left(x_{i}\right)}\left(1-F\left(x_{n}\right)\right)^{k-1} f\left(x_{n}\right), & x_{1}<\cdots<x_{n}, \\
0 & \text { otherwise. }\end{cases}
\end{aligned}
$$

Hence the probability density functions of $Y_{n}^{(k)}$ and $\left(Y_{m}^{(k)}, Y_{n}^{(k)}\right), m<n$, have the following forms:

$$
f_{Y_{n}^{(k)}}(x)=\frac{k^{n}}{(n-1) !}(-\ln (1-F(x)))^{n-1}(1-F(x))^{k-1} f(x), \quad n \geq 1,
$$

and

$$
\begin{aligned}
& f_{Y_{m}^{(k)}, Y_{n}^{(k)}}(x, y)=\frac{k^{n}}{(m-1) !(n-m-1) !}(\ln (1-F(x))-\ln (1-F(y)))^{n-m-1} \\
& \times(-\ln (1-F(x)))^{m-1} \frac{f(x)}{1-F(x)}(1-F(y))^{k-1} f(y), \quad x<y, n \geq 2 .
\end{aligned}
$$

Now we define the sequence $\left\{L_{k}(n), n \geq 1\right\}$ of $k$ th lower record times:

$$
\begin{aligned}
L_{k}(1) & =1, \\
L_{k}(n+1) & =\min \left\{j>L_{k}(n): X_{k: L_{k}(n)+k-1}>X_{k: j+k-1}\right\}, \quad n \geq 1 .
\end{aligned}
$$


The sequence $\left\{Z_{n}^{(k)}, n \geq 1\right\}$ with $Z_{n}^{(k)}=X_{k: L_{k}(n)+k-1}$, is called the sequence of $k$ th lower record values of $\left\{X_{n}, n \geq 1\right\}$. The probability density function of $\left(Z_{1}^{(k)}, \ldots, Z_{n}^{(k)}\right)$ has the form

$$
\begin{aligned}
& f_{Z_{1}^{(k)}, \ldots, Z_{n}^{(k)}}\left(x_{1}, \ldots, x_{n}\right) \\
&= \begin{cases}k^{n} \prod_{i=1}^{n-1} \frac{f\left(x_{i}\right)}{F\left(x_{i}\right)}\left(F\left(x_{n}\right)\right)^{k-1} f\left(x_{n}\right), & x_{1}<\cdots<x_{n}, \\
0 & \text { otherwise. }\end{cases}
\end{aligned}
$$

Hence the pdf's of $Z_{n}^{(k)}$ and $\left(Z_{m}^{(k)}, Z_{n}^{(k)}\right), m<n$, are as follows:

$$
\begin{aligned}
f_{Z_{n}^{(k)}}(x)= & \frac{k^{n}}{(n-1) !}(-\ln (F(x)))^{n-1}(F(x))^{k-1} f(x), \quad n \geq 1, \\
f_{Z_{m}^{(k)}, Z_{n}^{(k)}}(x, y)= & \frac{k^{n}}{(m-1) !(n-m-1) !}(\ln (F(x))-\ln (F(y)))^{n-m-1} \\
& \times(-\ln (F(x)))^{m-1} \frac{f(x)}{F(x)}(F(y))^{k-1} f(y), \quad x>y, \quad n \geq 2,
\end{aligned}
$$

respectively.

2. The least-squares estimators of $\mu$ and $\sigma$ using $k$ th record values. The use of order statistics in the estimation of parameters was presented in [8]. Our approach to the estimation of the location and scale parameters $\mu$ and $\sigma$ of a variate $X$ whose distribution depends on only these two parameters is based on the $k$ th record values.

Let $\left\{X_{n}, n \geq 1\right\}$ be a sequence of independent observations of $X$ and

$$
{ }^{*} X_{n}=\frac{X_{n}-\mu}{\sigma}, \quad n=1,2, \ldots,
$$

denote the standardized variants which may be regarded as independent observations of the standardized variate

$$
{ }^{*} X=\frac{X-\mu}{\sigma} .
$$

Let $Y_{1}^{(k)}, \ldots, Y_{m}^{(k)}$ be the first $m$ of the $k$ th upper record values from $\left\{X_{n}\right.$, $n \geq 1\}$ and $Z_{1}^{(k)}, \ldots, Z_{m}^{(k)}$ be the first $m$ of the $k$ th lower record values. Then

$$
{ }^{*} Y_{i}^{(k)}=\frac{Y_{i}^{(k)}-\mu}{\sigma}, \quad{ }^{*} Z_{i}^{(k)}=\frac{Z_{i}^{(k)}-\mu}{\sigma}, \quad i=1, \ldots, m,
$$

are the sequences of $k$ th upper and lower record values based on $\left\{{ }^{*} X_{n}, n \geq 1\right\}$. 
Write

$$
\begin{gathered}
\alpha_{i}:=\mathrm{E}\left[{ }^{*} Y_{i}^{(k)}\right], \quad \xi_{i}:=\mathrm{E}\left[{ }^{*} Z_{i}^{(k)}\right], \\
\omega_{i i}:=\operatorname{Var}\left[{ }^{*} Y_{i}^{(k)}\right], \quad \psi_{i i}:=\operatorname{Var}\left[{ }^{*} Z_{i}^{(k)}\right], \\
\omega_{i j}:=\operatorname{Cov}\left[{ }^{*} Y_{i}^{(k)},{ }^{*} Y_{j}^{(k)}\right], \quad \psi_{i j}:=\operatorname{Cov}\left[{ }^{*} Z_{i}^{(k)},{ }^{*} Z_{j}^{(k)}\right], \quad i, j=1, \ldots, m ; i<j .
\end{gathered}
$$

Reverting now to the original observations we have

$$
\begin{array}{ll}
\mathrm{E}\left[Y_{i}^{(k)}\right]=\mu+\sigma \alpha_{i}, & \mathrm{E}\left[Z_{i}^{(k)}\right]=\mu+\sigma \xi_{i}, \\
\operatorname{Var}\left[Y_{i}^{(k)}\right]=\sigma^{2} \omega_{i i}, & \operatorname{Var}\left[Z_{i}^{(k)}\right]=\sigma^{2} \psi_{i i}, \\
\operatorname{Cov}\left[Y_{i}^{(k)}, Y_{j}^{(k)}\right]=\sigma^{2} \omega_{i j}, & \operatorname{Cov}\left[Z_{i}^{(k)}, Z_{j}^{(k)}\right]=\sigma^{2} \psi_{i j} .
\end{array}
$$

We see that $\mathrm{E}\left[Y_{i}^{(k)}\right]$ (resp. $\mathrm{E}\left[Z_{i}^{(k)}\right]$ ) are linear functions of the parameters $\mu$ and $\sigma$ with known coefficients $\alpha_{i}$ (resp. $\xi_{i}$ ), and $\operatorname{Var}\left[Y_{i}^{(k)}\right]=\sigma^{2} \omega_{i i}$ (resp. $\left.\operatorname{Var}\left[Z_{i}^{(k)}\right]=\sigma^{2} \psi_{i i}\right)$ and $\operatorname{Cov}\left[Y_{i}^{(k)}, Y_{j}^{(k)}\right]=\sigma^{2} \omega_{i j}\left(\operatorname{resp} . \operatorname{Cov}\left[Z_{i}^{(k)}, Z_{j}^{(k)}\right]=\right.$ $\sigma^{2} \psi_{i j}$ ) are known up to a scale factor $\sigma^{2}$. The least-squares theorem of Gauss and Markov (cf. [13]) will be applied to derive the unbiased linear estimators of $\mu$ and $\sigma$ with minimal variance. We write the above results (5) in matrix form, as follows:

$$
\mathrm{E} \mathbf{Y}=\mu \mathbf{1}+\sigma \boldsymbol{\alpha}, \quad \mathrm{E} \mathbf{Z}=\mu \mathbf{1}+\sigma \boldsymbol{\xi},
$$

where $\mathbf{Y}$ is the (column) vector of the $Y_{i}^{(k)}$ and $\mathbf{Z}$ is the vector of the $Z_{i}^{(k)}, \boldsymbol{\alpha}$ the vector of the $\alpha_{i}, \boldsymbol{\xi}$ the vector of the $\xi_{i}$, and $\mathbf{1}$ a vector with unit elements. The equation (6) can be written as follows:

$$
\mathrm{E} \mathbf{Y}=\mathbf{p} \Theta, \quad \mathrm{E} \mathbf{Z}=\mathbf{p} \Theta,
$$

where $\mathbf{p}$ is the $m \times 2$ matrix $(\mathbf{1}, \boldsymbol{\alpha})$ or $(\mathbf{1}, \boldsymbol{\xi})$ and $\boldsymbol{\Theta}^{\prime}=(\mu, \sigma)$. The variance matrices of the $Y_{i}^{(k)}$ and $Z_{i}^{(k)}$, i.e. the matrices of variances and covariances, are

$$
\mathrm{V}(\mathbf{Y})=\sigma^{2} \boldsymbol{\omega}, \quad \mathrm{V}(\mathbf{Z})=\sigma^{2} \boldsymbol{\psi},
$$

where $\boldsymbol{\omega}$ and $\boldsymbol{\psi}$ are the $m \times m$ symmetric positive-definite matrices of all the $\omega_{i j}$ and $\psi_{i j}$. From the theorem of Gauss and Markov the required estimators $\widehat{\theta}_{1}, \widehat{\theta}_{2}$ of the vector $\boldsymbol{\Theta}$ are given by

$$
\widehat{\theta}_{1}=\left(\mathbf{p}^{\prime} \mathbf{\Omega} \mathbf{p}\right)^{-1} \mathbf{p}^{\prime} \mathbf{\Omega} \mathbf{Y}, \quad \widehat{\theta}_{2}=\left(\mathbf{p}^{\prime} \mathbf{\Psi} \mathbf{p}\right)^{-1} \mathbf{p}^{\prime} \mathbf{\Psi} \mathbf{Z},
$$

where $\boldsymbol{\Omega}=\boldsymbol{\omega}^{-1}$ and $\boldsymbol{\Psi}=\boldsymbol{\psi}^{-1}$. The variance matrices of the estimates are $\left(\mathbf{p}^{\prime} \boldsymbol{\Omega} \mathbf{p}\right)^{-1} \sigma^{2},\left(\mathbf{p}^{\prime} \mathbf{\Psi} \mathbf{p}\right)^{-1} \sigma^{2}$ where

$$
p^{\prime} \Omega p=\left[\begin{array}{cc}
1^{\prime} \Omega 1 & 1^{\prime} \Omega \alpha \\
1^{\prime} \Omega \alpha & \alpha^{\prime} \Omega \alpha
\end{array}\right], \quad p^{\prime} \Psi p=\left[\begin{array}{cc}
1^{\prime} \Psi 1 & 1^{\prime} \Psi \xi \\
1^{\prime} \Psi \xi & \xi^{\prime} \Psi \xi
\end{array}\right]
$$


the elements of these matrices being, of course, scalars. The inverses of these matrices are

$$
\left(\mathbf{p}^{\prime} \boldsymbol{\Omega} \mathbf{p}\right)^{-1}=\frac{1}{\triangle}\left[\begin{array}{cc}
\boldsymbol{\alpha}^{\prime} \boldsymbol{\Omega} \boldsymbol{\alpha} & -\mathbf{1}^{\prime} \boldsymbol{\Omega} \boldsymbol{\alpha} \\
-\mathbf{1}^{\prime} \boldsymbol{\Omega} \boldsymbol{\alpha} & \mathbf{1}^{\prime} \boldsymbol{\Omega} \mathbf{1}
\end{array}\right], \quad\left(\mathbf{p}^{\prime} \boldsymbol{\Psi} \mathbf{p}\right)^{-1}=\frac{1}{\triangle}\left[\begin{array}{cc}
\boldsymbol{\xi}^{\prime} \boldsymbol{\Psi} & -\mathbf{1}^{\prime} \boldsymbol{\Psi} \boldsymbol{c} \\
-\mathbf{1}^{\prime} \boldsymbol{\Psi} \boldsymbol{\xi} & \mathbf{1}^{\prime} \boldsymbol{\Psi} \mathbf{1}
\end{array}\right],
$$

where $\triangle$ is the determinant of the matrix $\mathbf{p}^{\prime} \boldsymbol{\Omega} \mathbf{p}$ or $\mathbf{p}^{\prime} \mathbf{\Psi} \mathbf{p}$, respectively.

Inserting the above quantities in (9) we get

$$
\begin{array}{ll}
\widehat{\mu}_{1}=-\boldsymbol{\alpha}^{\prime} \boldsymbol{\Gamma} \mathbf{Y}, & \widehat{\mu}_{2}=-\boldsymbol{\xi}^{\prime} \Upsilon \mathbf{Z}, \\
\widehat{\sigma}_{1}=\mathbf{1}^{\prime} \boldsymbol{\Gamma} \mathbf{Y}, & \widehat{\sigma}_{2}=\mathbf{1}^{\prime} \Upsilon \mathbf{Z},
\end{array}
$$

where $\boldsymbol{\Gamma}$ and $\Upsilon$ are the symmetric matrices defined by

$$
\Gamma=\frac{\Omega\left(1 \alpha^{\prime}-\alpha 1^{\prime}\right) \Omega}{\triangle}, \quad \Upsilon=\frac{\Psi\left(1 \xi^{\prime}-\xi 1^{\prime}\right) \Psi}{\triangle} .
$$

The variance and covariance of these estimates are given by

$$
\begin{array}{ll}
\operatorname{Var}\left(\widehat{\mu}_{1}\right)=\sigma^{2} \frac{\boldsymbol{\alpha}^{\prime} \boldsymbol{\Omega} \boldsymbol{\alpha}}{\triangle}, & \operatorname{Var}\left(\widehat{\mu}_{2}\right)=\sigma^{2} \frac{\boldsymbol{\xi}^{\prime} \boldsymbol{\Psi} \boldsymbol{\xi}}{\triangle}, \\
\operatorname{Var}\left(\widehat{\sigma}_{1}\right)=\sigma^{2} \frac{\mathbf{1}^{\prime} \boldsymbol{\Omega} \mathbf{1}}{\triangle}, & \operatorname{Var}\left(\widehat{\sigma}_{2}\right)=\sigma^{2} \frac{\mathbf{1}^{\prime} \boldsymbol{\Psi} \mathbf{1}}{\triangle}, \\
\operatorname{Cov}\left(\widehat{\mu}_{1}, \widehat{\sigma}_{1}\right)=-\sigma^{2} \frac{\mathbf{1}^{\prime} \boldsymbol{\Omega} \boldsymbol{\alpha}}{\triangle}, & \operatorname{Cov}\left(\widehat{\mu}_{2}, \widehat{\sigma}_{2}\right)=-\sigma^{2} \frac{\mathbf{1}^{\prime} \boldsymbol{\Psi} \boldsymbol{\xi}}{\triangle} .
\end{array}
$$

\section{Estimators of parameters for the Gumbel distribution}

3.1. Minimum variance linear unbiased estimators (MVLUE)

THEOREM 1. The minimum variance linear unbiased estimators $\widehat{\mu}_{G M}^{(k)}$ and $\widehat{\sigma}_{G M}^{(k)}$ of the Gumbel parameters $\mu$ and $\sigma$ based on the observed kth lower record values $z_{1}^{(k)}, z_{2}^{(k)}, \ldots, z_{m}^{(k)}$ are

$$
\begin{aligned}
& \widehat{\mu}_{G M}^{(k)}=z_{m}^{(k)}-(\nu(m)+\ln k)\left[(m-1)^{-1} \sum_{i=1}^{m-1} z_{i}^{(k)}-z_{m}^{(k)}\right], \\
& \widehat{\sigma}_{G M}^{(k)}=(m-1)^{-1} \sum_{i=1}^{m-1} z_{i}^{(k)}-z_{m}^{(k)} .
\end{aligned}
$$

The corresponding variances and covariance are

$$
\begin{aligned}
& \operatorname{Var}\left(\widehat{\mu}_{G M}^{(k)}\right)=\sigma^{2}\left(\frac{(\nu(m)+\ln k)^{2}}{(m-1)}+V_{m, m}^{*}\right), \quad \operatorname{Var}\left(\widehat{\sigma}_{G M}^{(k)}\right)=\frac{\sigma^{2}}{m-1}, \\
& \operatorname{Cov}\left(\widehat{\mu}_{G M}^{(k)}, \widehat{\sigma}_{G M}^{(k)}\right)=-\frac{\sigma^{2}(\nu(m)+\ln k)}{m-1},
\end{aligned}
$$


where

$$
\begin{aligned}
& \nu(1)=\gamma, \quad \nu(i)=\nu(i-1)-(i-1)^{-1}, \quad i \geq 2, \\
& V_{1,1}^{*}=\frac{\pi^{2}}{6}, \quad V_{i, i}^{*}=V_{i-1, i-1}^{*}-(i-1)^{-2}, \quad i \geq 2,
\end{aligned}
$$

where $\gamma$ is the Euler constant $(\gamma=0.57722)$.

Proof. We see that

$$
\begin{aligned}
& \mathrm{E}\left(Z_{i}^{(k)}\right)=\mu+\xi_{i}^{(k)} \sigma, \quad \xi_{i}^{(k)}=\ln k+\nu(i), \quad i=1, \ldots, m, \\
& \operatorname{Var}\left(Z_{i}^{(k)}\right)=\sigma^{2} V_{i, i}^{*}, \\
& \operatorname{Cov}\left(Z_{i}^{(k)}, Z_{j}^{(k)}\right)=\operatorname{Var}\left(Z_{j}^{(k)}\right), \quad i<j \quad(\text { cf. [11]). }
\end{aligned}
$$

Let $\boldsymbol{\Psi}(m \times m)=\left[\psi^{i j}\right]$. Then

$$
\begin{aligned}
& \psi^{i i}=i^{2}+(i-1)^{2}, \quad i=1, \ldots, m-1, \\
& \psi^{i j}= \begin{cases}(-1)^{i+j} \min \left(i^{2}, j^{2}\right), & i \neq j,|i-j|=1, \\
0, & |i-j|>1,\end{cases} \\
& \psi^{m m}=(m-1)^{2}+\frac{1}{V_{m, m}^{*}},
\end{aligned}
$$

and

$$
\begin{aligned}
& \mathbf{1}^{\prime} \boldsymbol{\Psi}=\left(0,0, \ldots, \frac{1}{V_{m, m}^{*}}\right), \quad \boldsymbol{\xi}^{\prime} \boldsymbol{\Psi}=\left(1,1, \ldots, \frac{\xi_{m}^{(k)}}{V_{m, m}^{*}}-(m-1)\right), \\
& \boldsymbol{\xi}^{\prime} \boldsymbol{\Psi} \mathbf{1}=\frac{\xi_{m}^{(k)}}{V_{m, m}^{*}}, \quad \boldsymbol{\xi}^{\prime} \boldsymbol{\Psi} \boldsymbol{\xi}=\frac{\left(\xi_{m}^{(k)}\right)^{2}}{V_{m, m}^{*}}+m-1, \quad \triangle=\frac{m-1}{V_{m, m}^{*}} .
\end{aligned}
$$

From (10), we see that $\widehat{\mu}_{G M}^{(k)}$ and $\widehat{\sigma}_{G M}^{(k)}$ are as in (12).

REMARK 1. For $k=1$ we obtain the estimators

$$
\begin{aligned}
& \widehat{\mu}_{G M}^{(1)}=z_{m}^{(1)}-\nu(m)\left[(m-1)^{-1} \sum_{i=1}^{m-1} z_{i}^{(1)}-z_{m}^{(1)}\right], \\
& \widehat{\sigma}_{G M}^{(1)}=(m-1)^{-1} \sum_{i=1}^{m-1} z_{i}^{(1)}-z_{m}^{(1)}
\end{aligned}
$$

(cf. [1], [2]).

REMARK 2. The Bayesian estimators of the parameters $\mu$ and $\sigma$ are given by 


$$
\begin{aligned}
\widehat{\mu}_{B}^{(k)} & =z_{m}^{(k)}-(\nu(m)+\ln k) \frac{m\left(\bar{z}^{(k)}-z_{m}^{(k)}\right)+\beta}{m+\alpha-1}, \\
\widehat{\sigma}_{B}^{(k)} & =\frac{m\left(\bar{z}^{(k)}-z_{m}^{(k)}\right)+\beta}{m+\alpha-1}
\end{aligned}
$$

where

$$
\bar{z}^{(k)}=\sum_{i=1}^{m} z_{i}^{(k)} / m,
$$

and $\alpha, \beta>0$ are the parameters of a prior distribution given by

$$
g(\mu, \sigma) \propto \frac{\beta^{\alpha}}{\Gamma(\alpha) \sigma^{\alpha+2}} e^{-\beta / \sigma}, \quad-\infty<\mu<\infty, \sigma>0,
$$

(cf. [9]). If $\alpha$ and $\beta$ in (14) tend to zero, then

$$
\begin{aligned}
& \widehat{\mu}_{B}^{(k)}=z_{m}^{(k)}-(\nu(m)+\ln k) \frac{m\left(\bar{z}^{(k)}-z_{m}^{(k)}\right)}{m-1}, \\
& \widehat{\sigma}_{B}^{(k)}=(m-1)^{-1} \sum_{i=1}^{m-1} z_{i}^{(k)}-z_{m}^{(k)}
\end{aligned}
$$

are the estimators $\widehat{\mu}_{G M}^{(k)}$ and $\widehat{\sigma}_{G M}^{(k)}$ given in (12), and the estimators $\widehat{\mu}_{B}^{(k)}$ and $\widehat{\sigma}_{B}^{(k)}$ for $k=1$ coincide with the estimators in terms of record values (cf. [6]).

3.2. Best linear invariant estimators (BLIE). In this section we present the best linear invariant estimators for the parameters of the Gumbel distribution. "Best" is used in the sense of minimum mean squared error and "invariant" with respect to the location parameter.

THEOREM 2. The best invariant estimators $\widetilde{\sigma}_{B L}^{(k)}$ and $\widetilde{\mu}_{B L}^{(k)}$ of the location and scale parameters of the Gumbel distribution (1) based on the first $m$ kth lower record values (BLIE) are

$$
\widetilde{\mu}_{B L}^{(k)}=\widehat{\mu}_{G M}^{(k)}-\widehat{\sigma}_{G M}^{(k)} \frac{(\nu(m)+\ln k)}{m}, \quad \widetilde{\sigma}_{B L}^{(k)}=\widehat{\sigma}_{G M}^{(k)} \frac{m-1}{m} .
$$

The mean squared errors of $\widetilde{\mu}_{B L}^{(k)}$ and $\widetilde{\sigma}_{B L}^{(k)}$ are

$$
\operatorname{MSE}\left(\widetilde{\mu}_{B L}^{(k)}\right)=\sigma^{2}\left[\frac{(\nu(m)+\ln k)^{2}}{m}+V_{m, m}^{*}\right], \quad \operatorname{MSE}\left(\widetilde{\sigma}_{B L}^{(k)}\right)=\frac{\sigma^{2}}{m},
$$

where $\widehat{\sigma}_{G M}^{(k)}$ and $\widehat{\mu}_{G M}^{(k)}$ are the MVLUE for $\sigma$ and $\mu$ given by (12).

Proof. Using the method of Mann (cf. [10]) we obtain

$$
\widetilde{\mu}_{B L}^{(k)}=\widehat{\mu}_{G M}^{(k)}-\widehat{\sigma}_{G M}^{(k)}\left[\mathrm{E}_{12}\left(1+\mathrm{E}_{22}\right)^{-1}\right], \quad \widetilde{\sigma}_{B L}^{(k)}=\widehat{\sigma}_{G M}^{(k)}\left(1+\mathrm{E}_{22}\right)^{-1},
$$


where $\mathrm{E}_{11}, \mathrm{E}_{12}$ and $\mathrm{E}_{22}$ are taken from

$$
\sigma^{2}\left[\begin{array}{ll}
\mathrm{E}_{11} & \mathrm{E}_{12} \\
\mathrm{E}_{12} & \mathrm{E}_{22}
\end{array}\right]:=\left[\begin{array}{cc}
\operatorname{Var}\left(\widehat{\mu}_{G M}^{(k)}\right) & \operatorname{Cov}\left(\widehat{\mu}_{G M}^{(k)}, \widehat{\sigma}_{G M}^{(k)}\right) \\
\operatorname{Cov}\left(\widehat{\mu}_{G M}^{(k)}, \widehat{\sigma}_{G M}^{(k)}\right) & \operatorname{Var}\left(\widehat{\sigma}_{G M}^{(k)}\right)
\end{array}\right],
$$

after using (13), i.e.

$$
\mathrm{E}_{11}=\frac{(\nu(m)+\ln k)^{2}}{m-1}+V_{m m}^{*}, \quad \mathrm{E}_{12}=-\frac{(\nu(m)+\ln k)}{m-1}, \quad \mathrm{E}_{22}=\frac{1}{m-1} .
$$

Moreover, we have

$$
\operatorname{MSE}\left(\widetilde{\mu}_{B L}^{(k)}\right)=\sigma^{2}\left[\mathrm{E}_{11}-\mathrm{E}_{12}^{2}\left(1+\mathrm{E}_{22}\right)^{-1}\right], \quad \operatorname{MSE}\left(\widetilde{\sigma}_{B L}^{(k)}\right)=\sigma^{2} \mathrm{E}_{22}\left(1+\mathrm{E}_{22}\right)^{-1} .
$$

REMARK 3. For $k=1$ the best linear invariant estimators are

(cf. [6])

$$
\widetilde{\mu}_{B L}^{(1)}=\widehat{\mu}_{G M}^{(1)}-\widehat{\sigma}_{G M}^{(1)} \frac{\nu(m)}{m}, \quad \widetilde{\sigma}_{B L}^{(1)}=\widehat{\sigma}_{G M}^{(1)} \frac{m-1}{m}
$$

3.3. Maximum likelihood estimators (MLE). We now give the maximum likelihood estimators for the parameters of the Gumbel distribution.

THEOREM 3. The maximum likelihood estimators $\widehat{\sigma}_{M L}^{(k)}$ and $\widehat{\mu}_{M L}^{(k)}$ of the location and scale parameters of the Gumbel distribution (1) based on the first $m$ kth lower record values are

$$
\widehat{\mu}_{M L}^{(k)}=z_{m}^{(k)}+\ln \left(\frac{m}{k}\right)\left[\bar{z}^{(k)}-z_{m}^{(k)}\right], \quad \widehat{\sigma}_{M L}^{(k)}=\bar{z}^{(k)}-z_{m}^{(k)},
$$

where

$$
\bar{z}^{(k)}=\sum_{i=1}^{m} z_{i}^{(k)} / m
$$

The variance and covariance of the estimators are

$$
\begin{aligned}
& \operatorname{Var}\left(\widehat{\mu}_{M L}^{(k)}\right)=\sigma^{2}\left(V_{m m}^{*}+\left(\frac{m-1}{m}\right)^{2} \frac{\left(\ln \left(\frac{m}{k}\right)\right)^{2}}{m}\right), \\
& \operatorname{Var}\left(\widehat{\sigma}_{M L}^{(k)}\right)=\left(\frac{m-1}{m}\right)^{2} \frac{\sigma^{2}}{m} .
\end{aligned}
$$

Proof. Using (4) we see that the likelihood function $L$ based on the $k$ th record values for the Gumbel distribution has the form

$$
\begin{aligned}
L\left(\mu, \sigma \mid \underline{z}^{(k)}\right)= & k^{m}\left(\prod_{i=1}^{m-1} \frac{f\left(z_{i}^{(k)}\right)}{F\left(z_{i}^{(k)}\right)}\right)\left[F\left(z_{m}^{(k)}\right)\right]^{k-1} f\left(z_{m}^{(k)}\right) \\
= & \frac{k^{m}}{\sigma^{m}} \exp \left[-m \frac{\bar{z}^{(k)}-\mu}{\sigma}-k \exp \left(-\frac{z_{m}^{(k)}-\mu}{\sigma}\right)\right], \\
& z_{1}^{(k)}>z_{2}^{(k)}>\cdots>z_{m}^{(k)}, \quad \underline{z}^{(k)}=\left(z_{1}^{(k)}, z_{2}^{(k)}, \ldots, z_{m}^{(k)}\right) .
\end{aligned}
$$


Hence we get

$\ln L\left(\mu, \sigma \mid \underline{z}^{(k)}\right)=\ln k^{m}-\ln \sigma^{m}+\left[-m\left(\frac{\bar{z}^{(k)}-\mu}{\sigma}\right)-k \exp \left(-\left(\frac{z_{m}^{(k)}-\mu}{\sigma}\right)\right)\right]$,

and

$$
\begin{gathered}
\frac{\partial \ln L\left(\mu, \sigma \mid \underline{z}^{(k)}\right)}{\partial \sigma}=-\frac{m}{\sigma}+\frac{m\left(\bar{z}^{(k)}-\mu\right)}{\sigma^{2}}-k \exp \left(-\left(\frac{z_{m}^{(k)}-\mu}{\sigma}\right)\right)\left(\frac{z_{m}^{(k)}-\mu}{\sigma^{2}}\right)=0, \\
\frac{\partial \ln L\left(\mu, \sigma \mid \underline{z}^{(k)}\right)}{\partial \mu}=\frac{m}{\sigma}-\frac{k}{\sigma} \exp \left(-\left(\frac{z_{m}^{(k)}-\mu}{\sigma}\right)\right)=0 .
\end{gathered}
$$

After standard evaluations we get the maximum likelihood estimators given by (16). The estimators $\widehat{\mu}_{M L}^{(k)}$ and $\widehat{\sigma}_{M L}^{(k)}$ are both biased. We see that

$$
\begin{aligned}
& \mathrm{E}\left(\widehat{\mu}_{M L}^{(k)}\right)=\mu+\left(\nu(m)+\ln k+\frac{m-1}{m} \ln \left(\frac{m}{k}\right)\right) \sigma, \\
& \mathrm{E}\left(\widehat{\sigma}_{M L}^{(k)}\right)=\frac{m-1}{m} \sigma .
\end{aligned}
$$

Table 1. The estimators MVLUE, BLIE, MLE and Bayes estimators, based on generated $k$ th record values, when the population parameters are $\sigma=1.0$ and $\mu=2.0$

\begin{tabular}{cccccccccc}
\hline$k$ & $m$ & $\widehat{\sigma}_{G M}^{(k)}$ & $\widehat{\mu}_{G M}^{(k)}$ & $\widetilde{\sigma}_{B L}^{(k)}$ & $\widetilde{\mu}_{B L}^{(k)}$ & $\widehat{\sigma}_{M L}^{(k)}$ & $\widehat{\mu}_{M L}^{(k)}$ & $\widehat{\sigma}_{B}^{(k)}$ & $\widehat{\mu}_{B}^{(k)}$ \\
\hline 1 & 4 & 1.4321 & 2.4929 & 1.0740 & 2.9426 & 1.0740 & 2.1830 & $1.2592^{a}$ & $2.2758^{a}$ \\
& & & & & & & & $1.2160^{b}$ & $2.2215^{b}$ \\
& 6 & 0.9375 & 2.2138 & 0.7813 & 2.4804 & 0.7813 & 2.0141 & $0.9554^{a}$ & $2.2442^{a}$ \\
& & & & & & & & $0.9609^{b}$ & $2.2538^{b}$ \\
\hline 2 & 4 & 1.1269 & 2.2117 & 0.8451 & 2.3703 & 0.8451 & 2.1631 & $1.0761^{a}$ & $2.1831^{a}$ \\
& & & & & & & & $1.0634^{b}$ & $2.1760^{b}$ \\
& 6 & 1.3854 & 2.1541 & 1.1545 & 2.3880 & 1.1545 & 2.0191 & $1.2753^{a}$ & $2.0426^{a}$ \\
& & & & & & & & $1.2409^{b}$ & $2.0077^{b}$ \\
\hline 3 & 4 & 1.0606 & 1.9118 & 0.7954 & 1.9538 & 0.7954 & 1.9736 & $1.0363^{a}$ & $1.9080^{a}$ \\
& & & & & & & & $1.0303^{b}$ & $1.9070^{b}$ \\
& 6 & 0.7998 & 2.0632 & 0.6665 & 2.1441 & 0.6665 & 2.0393 & $0.8570^{a}$ & $2.0979^{a}$ \\
& & & & & & & & $0.8748^{b}$ & $2.1088^{b}$ \\
\hline 5 & 4 & 1.0715 & 2.2093 & 0.8036 & 2.1147 & 0.8036 & 2.4086 & $1.0429^{a}$ & $2.2195^{a}$ \\
& & & & & & & & $1.0357^{b}$ & $2.2220^{b}$ \\
& 6 & 1.3782 & 1.8780 & 1.1485 & 1.9002 & 1.1485 & 1.9541 & $1.2018^{a}$ & $1.8675^{a}$ \\
& & & & & & & $1.2364^{b}$ & $1.8643^{b}$ \\
\hline
\end{tabular}

${ }^{a}$ When the prior parameters are both equal to 2.0 .

${ }^{b}$ When the prior parameters are both equal to 3.0. 
REMARK 4. For $k=1$ we have

$$
\widehat{\mu}_{M L}^{(1)}=z_{m}^{(1)}+\ln (m)\left[\bar{z}^{(1)}-z_{m}^{(1)}\right], \quad \widehat{\sigma}_{M L}^{(1)}=\bar{z}^{(1)}-z_{m}^{(1)},
$$

which are the maximum likelihood estimators given in [1] and [2].

3.4. Numerical illustration. In order to illustrate the usefulness of the estimators discussed in Section 3, simulated $k$ th record sets of sizes $m=4$ and 6 for $k=1,2,3$ and 5 from the Gumbel distribution with parameters $\sigma=1.0$ and $\mu=2.0$ are obtained. The MVLUE, BLIE, MLE and Bayesian estimators of the parameters $\sigma$ and $\mu$, given respectively by (12), (15), (16) and (14) are calculated. Two pairs of prior parameters $(\alpha=2.0, \beta=2.0)$ and $(\alpha=3.0, \beta=3.0)$ are considered here. All the results are presented in Table 1.

\section{Estimators of parameters for the Burr distribution}

4.1. Minimum variance linear unbiased estimators (MVLUE). Here we consider the estimation of the location parameter $\mu$ and scale parameter $\sigma$ for the Burr distribution when the parameters $\lambda$ and $\beta$ are known. We need the following

LEMma 1. Let $\left\{X_{n}, n \geq 1\right\}$ be a sequence of iid random variables from the Burr distribution given by $(2)$ and let $\left(Y_{1}^{(k)}, \ldots, Y_{m}^{(k)}\right)$ denote a vector of $k$ th upper record values from $\left\{X_{n}\right\}$. Write

$$
c_{i}=k^{i} \lambda^{i}, \quad d_{i}=(k \lambda-1)^{i}, \quad e_{i}=(k \lambda-2)^{i} .
$$

Then

$$
\begin{aligned}
& \mathrm{E}\left[Y_{i}^{(k)}\right]=\mu+\alpha_{i} \sigma, \\
& \operatorname{Var}\left[Y_{i}^{(k)}\right]=\sigma^{2}\left(a_{i}-b_{i}\right) b_{i}, \\
& \operatorname{Cov}\left[Y_{i}^{(k)} Y_{j}^{(k)}\right]=\sigma^{2}\left(a_{i}-b_{i}\right) b_{j},
\end{aligned}
$$

where $\alpha_{i}=\beta\left(c_{i} / d_{i}-1\right), a_{i}=\beta d_{i} / e_{i}$ and $b_{i}=\beta c_{i} / d_{i}$.

Proof. We consider the Burr distribution in the form

$$
F(x)=1-\beta^{\lambda}(\beta+x)^{-\lambda}, \quad x>0 ; \quad \beta>0, \lambda>0 .
$$

The probability density function is given by

$$
f(x)=\lambda \beta^{\lambda}(\beta+x)^{-(\lambda+1)}, \quad x>0 ; \quad \beta>0, \lambda>0 .
$$


Let ${ }^{*} Y_{1}^{(k)}, \ldots,{ }^{*} Y_{m}^{(k)}$ be the first $m$ of $k$ th upper record values of $\left\{{ }^{*} X_{n}, n \geq 1\right\}$ from the Burr distribution given by (18). Then

$\mathrm{E}\left[{ }^{*} Y_{i}^{(k)}\right]$

$$
=\int_{0}^{\infty} \frac{k^{i}}{\Gamma(i)} y\left[-\ln \left(\beta^{\lambda}(\beta+y)^{-\lambda}\right)\right]^{i-1}\left(\beta^{\lambda}(\beta+y)^{-\lambda}\right)^{k-1} \lambda \beta^{\lambda}(\beta+y)^{-(\lambda+1)} d y,
$$

which after substitution $t=\beta^{\lambda}(\beta+y)^{-\lambda}$ gives

$$
\mathrm{E}\left[{ }^{*} Y_{i}^{(k)}\right]=\int_{0}^{1} \frac{k^{i}}{\Gamma(i)}\left(-\beta+\beta t^{-1 / \lambda}\right)[-\ln t]^{i-1} t^{k-1} d t=\beta\left(\frac{c_{i}}{d_{i}}-1\right)=\alpha_{i} .
$$

Then by (5) we have

$$
\mathrm{E}\left[Y_{i}^{(k)}\right]=\mu+\alpha_{i} \sigma .
$$

Similarly it can be shown that

$$
\mathrm{E}\left[{ }^{*} Y_{i}^{(k)}\right]^{2}=\beta^{2}\left(1-2 \frac{c_{i}}{d_{i}}+\frac{c_{i}}{e_{i}}\right) \quad \text { for } i=1, \ldots, m .
$$

Thus

$$
\operatorname{Var}\left[{ }^{*} Y_{i}^{(k)}\right]=\left(a_{i}-b_{i}\right) b_{i},
$$

and by (5) we obtain

$$
\operatorname{Var}\left[Y_{i}^{(k)}\right]=\sigma^{2}\left(a_{i}-b_{i}\right) b_{i}, \quad i=1, \ldots, m .
$$

Now we know that

$$
\begin{aligned}
\mathrm{E}\left(\left[{ }^{*} Y_{i}^{(k)}\right]\left[{ }^{*} Y_{j}^{(k)}\right]\right)= & {\left[\frac{k \lambda}{k \lambda-1}\right]^{j-i} \mathrm{E}\left[{ }^{*} Y_{i}^{(k)}\right]^{2} } \\
& +\frac{\beta}{k \lambda-1} \sum_{p=i+1}^{j}\left[\frac{k \lambda}{k \lambda-1}\right]^{j-p} \mathrm{E}\left[{ }^{*} Y_{i}^{(k)}\right], \\
\mathrm{E}\left[{ }^{*} Y_{j}^{(k)}\right]= & {\left[\frac{k \lambda}{k \lambda-1}\right]^{j-i} \mathrm{E}\left[{ }^{*} Y_{i}^{(k)}\right]+\frac{\beta}{k \lambda-1} \sum_{p=i+1}^{j}\left[\frac{k \lambda}{k \lambda-1}\right]^{j-p} }
\end{aligned}
$$

(cf. [12, Theorems 2.1 and 3.1]). Hence

$$
\operatorname{Cov}\left[{ }^{*} Y_{i}^{(k)},{ }^{*} Y_{j}^{(k)}\right]=\left[\frac{k \lambda}{k \lambda-1}\right]^{j-i} \mathrm{E}\left[{ }^{*} Y_{i}^{(k)}\right]^{2}-\left[\frac{k \lambda}{k \lambda-1}\right]^{j-i}\left(\mathrm{E}\left[{ }^{*} Y_{i}^{(k)}\right]\right)^{2},
$$

thus

$$
\operatorname{Cov}\left[{ }^{*} Y_{i}^{(k)},{ }^{*} Y_{j}^{(k)}\right]=\frac{c_{j}}{c_{i}} \frac{d_{i}}{d_{j}} \operatorname{Var}\left[{ }^{*} Y_{i}^{(k)}\right]=\left(a_{i}-b_{i}\right) b_{j}
$$


and by (5),

$$
\operatorname{Cov}\left[Y_{i}^{(k)}, Y_{j}^{(k)}\right]=\sigma^{2}\left(a_{i}-b_{i}\right) b_{j}, \quad i, j=1, \ldots, m .
$$

THEOREM 4. The minimum variance linear unbiased estimators $\widehat{\mu}_{G M}^{(k)}$ and $\widehat{\sigma}_{G M}^{(k)}$ for $\mu$ and $\sigma$ of the Burr distribution, based on the observed kth upper record values $y_{1}^{(k)}, \ldots, y_{m}^{(k)}$ are

$$
\widehat{\mu}_{G M}^{(k)}=\left[\sum_{i=1}^{m} w_{1 i} y_{i}^{(k)}\right], \quad \widehat{\sigma}_{G M}^{(k)}=\frac{1}{\beta} d_{1}\left(y_{1}^{(k)}-\widehat{\mu}_{G M}^{(k)}\right),
$$

where

$$
\begin{aligned}
w_{11} & =\frac{1}{D_{0}}\left\{T_{0} e_{1} d_{1}-\frac{e_{1}^{2}}{c_{1}}\right\} \\
w_{1 i} & =\frac{1}{D_{0}}\left\{-\frac{e_{i+1}}{c_{i}}\right\}, \quad i=2, \ldots, m-1, \\
w_{1 m} & =-\frac{1}{D_{0}} d_{1} \frac{e_{m+1}}{c_{m}}, \\
D_{0} & =e_{1} c_{1} T_{0}-e_{1}^{2}, \quad T_{0}=\sum_{i=1}^{m} \frac{e_{i}}{c_{i}} .
\end{aligned}
$$

with $e_{i}, c_{i}, d_{i}$ given in Lemma 1 . The variances and covariance of the estimators are

$$
\begin{aligned}
& \operatorname{Var}\left(\widehat{\mu}_{G M}^{(k)}\right)=\sigma^{2} \beta^{2} \frac{T_{0}}{D_{0}}, \quad \operatorname{Var}\left(\widehat{\sigma}_{G M}^{(k)}\right)=\sigma^{2} \frac{T_{0}+e_{1}^{2}}{D_{0}}, \\
& \operatorname{Cov}\left(\widehat{\mu}_{G M}^{(k)}, \widehat{\sigma}_{G M}^{(k)}\right)=\sigma^{2} \beta \frac{T_{0}-e_{1}}{D_{0}} .
\end{aligned}
$$

Proof. Let $\boldsymbol{Y}^{\prime}=\left(Y_{1}^{(k)}, \ldots, Y_{m}^{(k)}\right)$ be the vector of $k$ th upper record values. Then

$$
\mathrm{E} \boldsymbol{Y}=\mu \mathbf{1}+\boldsymbol{\alpha} \sigma,
$$

where $\boldsymbol{\alpha}^{\prime}=\left(\alpha_{1}, \ldots, \alpha_{m}\right)$ with $\alpha_{i}=\beta\left(c_{i} / d_{i}-1\right)$. We note that $\mathrm{E} \boldsymbol{Y}$ can be written as

$$
\mathrm{E} \mathbf{Y}=\mathbf{p} \Theta
$$

where $\mathbf{p}$ is the $m \times 2$ matrix $(\mathbf{1}, \boldsymbol{\alpha})$ and $\boldsymbol{\Theta}^{\prime}=(\mu, \sigma)$ (see (7)). The variance matrix of $\boldsymbol{Y}$ has the form given by (8) with

$$
\boldsymbol{\omega}(m \times m)=\left[\omega_{i j}\right]=\left[\left(a_{i}-b_{i}\right) b_{j}\right], \quad \text { where } \quad a_{i}=\beta \frac{d_{i}}{e_{i}}, b_{i}=\beta \frac{c_{i}}{d_{i}} .
$$

So we consider the general linear model of Gauss-Markov (cf. [13, pp. 122123]). 
Here the linear unbiased estimators with minimum variance $\widehat{\boldsymbol{\Theta}}^{\prime}=(\widehat{\mu}, \widehat{\sigma})$ of $\Theta^{\prime}$, using the Gauss-Markov theorem (cf. [13]) can be written as follows:

$$
\widehat{\boldsymbol{\Theta}}=\left(\begin{array}{c}
\widehat{\mu} \\
\widehat{\sigma}
\end{array}\right)=\left(\boldsymbol{B}^{\prime} \boldsymbol{B}\right)^{-1} \boldsymbol{B}^{\prime} \boldsymbol{U}=\boldsymbol{W} \boldsymbol{Y},
$$

where $\boldsymbol{U}:=\left(\boldsymbol{T}^{\prime}\right)^{-1} \boldsymbol{Y}, \boldsymbol{B}:=\left(\boldsymbol{T}^{\prime}\right)^{-1} \boldsymbol{p}, \boldsymbol{T}$ is the matrix such that $\boldsymbol{\omega}=\boldsymbol{T}^{\prime} \boldsymbol{T}$ and $\boldsymbol{W}:=\left(\boldsymbol{B}^{\prime} \boldsymbol{B}\right)^{-1} \boldsymbol{B}^{\prime}\left(\boldsymbol{T}^{\prime}\right)^{-1}$ (cf. [13]), i.e.

$$
\boldsymbol{W}=\left[\begin{array}{llll}
w_{11} & w_{12} & \ldots & w_{1 m} \\
w_{21} & w_{22} & \ldots & w_{2 m}
\end{array}\right] .
$$

Since $\boldsymbol{\omega}$ is positive definite, there exists an $m \times m$ matrix $\boldsymbol{T}$ such that $\boldsymbol{\omega}=$ $\boldsymbol{T} \boldsymbol{T}^{\prime}$. Using Cholesky's decomposition of $\boldsymbol{\omega}$ we get

$$
\boldsymbol{T}^{\prime}=\left[\begin{array}{cccc}
t_{11} & 0 & \ldots & 0 \\
t_{12} & t_{22} & \ldots & 0 \\
\ldots \ldots & \ldots & \ldots & \ldots \ldots \\
t_{1 m} & t_{2 m} & \ldots & t_{m m}
\end{array}\right],
$$

where $t_{11}=\sqrt{\omega_{11}}, t_{1 j}=\omega_{1 j} / t_{11}, j=2, \ldots, m$,

$$
\begin{aligned}
& t_{i i}=\sqrt{\omega_{i i}-\sum_{p=1}^{i-1} t_{p i}^{2}}, \quad t_{i j}=\frac{1}{t_{i i}}\left[\omega_{i j}-\sum_{p=1}^{i-1} t_{p i} t_{p j}\right], \quad i>j, \\
& t_{i j}=0, \quad j>i, i=2, \ldots, m .
\end{aligned}
$$

Hence $\left(\boldsymbol{T}^{\prime}\right)^{-1}$ has the form

$$
\left(\boldsymbol{T}^{\prime}\right)^{-1}=\left[\begin{array}{cccccc}
a_{11} & 0 & 0 & \ldots & 0 & 0 \\
a_{21} & a_{22} & 0 & \ldots & 0 & 0 \\
0 & a_{32} & a_{33} & \ldots & 0 & 0 \\
\ldots \ldots & \ldots & \ldots \ldots \ldots \ldots \ldots \ldots \ldots & \ldots \ldots \\
0 & 0 & 0 & \ldots & a_{m, m-1} & a_{m m}
\end{array}\right],
$$

where

$$
\begin{aligned}
& a_{11}=\frac{d_{1}}{\beta} \sqrt{\frac{e_{1}}{c_{1}}}, \quad a_{i i}=\frac{d_{1}}{\beta} \sqrt{\frac{e_{i}}{c_{i}}}, \\
& a_{i, i-1}=-\frac{c_{1}}{\beta} \sqrt{\frac{e_{i}}{c_{i}}}, \quad i=2, \ldots, m .
\end{aligned}
$$

Then we have $\mathrm{E}(\boldsymbol{U})=\boldsymbol{B} \Theta$ with

$$
\boldsymbol{B}^{\prime}=\left[\begin{array}{llll}
b_{11} & b_{21} & \ldots & b_{m 1} \\
b_{12} & b_{22} & \ldots & b_{m 2}
\end{array}\right],
$$


where

$$
\begin{aligned}
& b_{11}=\frac{d_{1}}{\beta} \sqrt{\frac{e_{1}}{c_{1}}}, \quad b_{i 1}=-\frac{1}{\beta} \sqrt{\frac{e_{i}}{c_{i}}}, \quad i=2, \ldots, m, \\
& b_{i 2}=\sqrt{\frac{e_{i}}{c_{i}}}, \quad i=1, \ldots, m .
\end{aligned}
$$

Therefore

$$
\left(\boldsymbol{B}^{\prime} \boldsymbol{B}\right)^{-1}=\frac{\beta^{2}}{D_{0}}\left[\begin{array}{cc}
T_{0} & \frac{T_{0}-e_{1}}{\beta} \\
\frac{T_{0}-e_{1}}{\beta} & \frac{T_{0}+e_{1}^{2}}{\beta^{2}}
\end{array}\right],
$$

where $T_{0}=\sum_{i=1}^{m} e_{i} / c_{i}$, and $D_{0}=e_{1} c_{1} T_{0}-e_{1}^{2}$.

From (24)-(26) we get the elements of $\boldsymbol{W}$ in (20) as follows:

$$
\begin{array}{rlrl}
w_{11} & =\frac{1}{D_{0}}\left\{T_{0} e_{1} d_{1}-\frac{e_{1}^{2}}{c_{1}}\right\}, & w_{1 i}=\frac{1}{D_{0}}\left\{-\frac{e_{i+1}}{c_{i}}\right\}, & i=2, \ldots, m-1, \\
w_{1 m}=-\frac{1}{D_{0}} d_{1} \frac{e_{m+1}}{c_{m}}, & w_{2 i}=-\frac{d_{1} w_{1 i}}{\beta}, & i=2, \ldots, m . \\
w_{21}=\frac{d_{1}}{\beta}-\frac{d_{1} w_{11}}{\beta}, &
\end{array}
$$

and by (22) we get the estimators (19).

The variance and covariance of the estimators are given by (cf. [13, p. 124])

$$
\operatorname{Var}\left(\widehat{\boldsymbol{\Theta}}^{\prime}\right)=\sigma^{2}\left(\boldsymbol{B}^{\prime} \boldsymbol{B}\right)^{-1}
$$

which by (25) proves (21).

COROLlary 1. When $\alpha$ is known, the minimum variance linear unbiased estimators for the parameters of the generalized Pareto distribution with the probability density function

$$
\begin{array}{ll}
f(x)=\frac{1}{\sigma}\left(1+\alpha \frac{x-\mu}{\sigma}\right)^{-\left(1+\alpha^{-1}\right)}, & x \geq \mu \\
& -\infty<\mu<\infty, \sigma>0, \alpha>0
\end{array}
$$

in terms of $k$ th record values, are given by

$$
\widehat{\mu}_{G M}^{(k)}=\left[\sum_{i=1}^{m} w_{1 i} y_{i}^{(k)}\right], \quad \widehat{\sigma}_{G M}^{(k)}=(k-\alpha)\left(y_{1}^{(k)}-\widehat{\mu}^{(k)}\right),
$$


where

$$
\begin{aligned}
w_{11} & =\frac{1}{D_{0}}\left\{T_{0} \frac{(k-2 \alpha)(k-\alpha)}{\alpha^{2}}-\frac{(k-2 \alpha)^{2}}{k \alpha}\right\}, \\
w_{1 i} & =-\frac{1}{D_{0}} \frac{(k-2 \alpha)^{i+1}}{k^{i} \alpha}, \quad i=2, \ldots, m-1, \\
w_{1 m} & =-\frac{1}{D_{0}} \frac{k-\alpha}{k^{m} \alpha^{2}}(k-2 \alpha)^{m+1}, \\
D_{0} & =\frac{k-2 \alpha}{\alpha^{2}}\left[k T_{0}-k+2 \alpha\right], \quad T_{0}=\sum_{i=1}^{m}\left(\frac{k-2 \alpha}{k}\right)^{i} .
\end{aligned}
$$

The variances and covariance of the estimators are given by

$$
\begin{aligned}
& \operatorname{Var}\left(\widehat{\mu}_{G M}^{(k)}\right)=\sigma^{2} \alpha^{-2} \frac{T_{0}}{D_{0}}, \quad \operatorname{Var}\left(\widehat{\sigma}_{G M}^{(k)}\right)=\sigma^{2} \frac{T_{0}+\left(\frac{k-2 \alpha}{\alpha}\right)^{2}}{D_{0}}, \\
& \operatorname{Cov}\left(\widehat{\mu}_{G M}^{(k)}, \widehat{\sigma}_{G M}^{(k)}\right)=\sigma^{2} \alpha^{-1} \frac{T_{0}-\frac{k-2 \alpha}{\alpha}}{D_{0}} .
\end{aligned}
$$

We obtain these estimators from (19) when $\lambda=\beta=\alpha^{-1}$.

REMARK 5. For $k=1$ the estimators $\widehat{\mu}_{G M}^{(k)}$ and $\widehat{\sigma}_{G M}^{(k)}$ coincide with those given by Ahsanullah (cf. [2]).

COROLlARY 2. When $\lambda$ is known, the minimum variance linear unbiased estimators for the parameters of the Lomax distribution with the probability density function

$$
f(x)=\frac{\lambda}{\sigma}\left(1+\frac{x-\mu}{\sigma}\right)^{-(\lambda+1)}, \quad x \geq \mu ; \quad-\infty<\mu<\infty, \sigma>0, \lambda>0,
$$

in terms of $k$ th record values are as follows:

$$
\widehat{\mu}_{G M}^{(k)}=\left[\sum_{i=1}^{m} w_{1 i} y_{i}^{(k)}\right], \quad \widehat{\sigma}_{G M}^{(k)}=\left(y_{1}^{(k)}-\widehat{\mu}_{G M}^{(k)}\right) d_{1},
$$

where

$$
\begin{aligned}
w_{11} & =\frac{1}{D_{0}}\left\{T_{0} e_{1} d_{1}-\frac{e_{1}^{2}}{c_{1}}\right\} \\
w_{1 i} & =\frac{1}{D_{0}}\left\{-\frac{e_{i+1}}{c_{i}}\right\}, \quad i=2, \ldots, m-1, \\
w_{1 m} & =-\frac{1}{D_{0}} d_{1} \frac{e_{m+1}}{c_{m}}, \\
D_{0} & =e_{1} c_{1} T_{0}-e_{1}^{2}, \quad T_{0}=\sum_{i=1}^{m} \frac{e_{i}}{c_{i}} .
\end{aligned}
$$


The variances and covariance of the estimators are

$$
\begin{aligned}
& \operatorname{Var}\left(\widehat{\mu}_{G M}^{(k)}\right)=\sigma^{2} \frac{T_{0}}{D_{0}}, \\
& \operatorname{Var}\left(\widehat{\sigma}_{G M}^{(k)}\right)=\sigma^{2} \frac{T_{0}+e_{1}^{2}}{D_{0}}, \\
& \operatorname{Cov}\left(\widehat{\mu}_{G M}^{(k)}, \widehat{\sigma}_{G M}^{(k)}\right)=\sigma^{2} \frac{T_{0}-e_{1}}{D_{0}} .
\end{aligned}
$$

We obtain these estimators from (19) when $\beta=1$.

REMARK 6 . The estimators $\widehat{\mu}_{G M}^{(k)}$ and $\widehat{\sigma}_{G M}^{(k)}$ for $k=1$ were given by Ahsanullah in [2].

4.2. Best linear invariant estimators (BLIE). We now consider the best invariant estimators for parameters of the Burr distribution when the parameters $\lambda$ and $\beta$ are known. "Best" is used in the sense of minimum mean squared error and "invariant" with respect to the location parameter.

THEOREM 5. The best invariant estimators $\widetilde{\mu}_{B L}^{(k)}$ and $\widetilde{\sigma}_{B L}^{(k)}$ of the location and scale parameters of the Burr distribution (2) based on the first $m$ kth upper record values (BLIE) are

$$
\widetilde{\mu}_{B L}^{(k)}=\widehat{\mu}_{G M}^{(k)}-\widehat{\sigma}_{G M}^{(k)}\left[\beta \frac{T_{0}-e_{1}}{T_{0}+D_{0}+e_{1}^{2}}\right], \quad \widetilde{\sigma}_{B L}^{(k)}=\widehat{\sigma}_{G M}^{(k)} \frac{D_{0}}{T_{0}+D_{0}+e_{1}^{2}} .
$$

The mean squared errors of $\widetilde{\mu}_{B L}^{(k)}$ and $\widetilde{\sigma}_{B L}^{(k)}$ are

$$
\begin{aligned}
\operatorname{MSE}\left(\widetilde{\mu}_{B L}^{(k)}\right) & =\sigma^{2} \beta^{2}\left[\frac{T_{0}}{D_{0}}-\beta \frac{\left(T_{0}-e_{1}\right)^{2}}{D_{0}\left(D_{0}+T_{0}+e_{1}^{2}\right)}\right], \\
\operatorname{MSE}\left(\widetilde{\sigma}_{B L}^{(k)}\right) & =\sigma^{2} \frac{T_{0}+e_{1}^{2}}{D_{0}+T_{0}+e_{1}^{2}},
\end{aligned}
$$

where $\widehat{\sigma}_{G M}^{(k)}$ and $\widehat{\mu}_{G M}^{(k)}$ are the MVLUE for $\sigma$ and $\mu$ given by (19), and $D_{0}=$ $e_{1} c_{1} T_{0}-e_{1}^{2}, T_{0}=\sum_{i=1}^{m} e_{i} / c_{i}$ with $e_{1}=(k \lambda-2)^{i}$.

Proof. Using the method of Mann (cf. [10]) we obtain

$$
\widetilde{\mu}_{B L}^{(k)}=\widehat{\mu}_{G M}^{(k)}-\widehat{\sigma}_{G M}^{(k)}\left[\mathrm{E}_{12}\left(1+\mathrm{E}_{22}\right)^{-1}\right], \quad \widetilde{\sigma}_{B L}^{(k)}=\widehat{\sigma}_{G M}^{(k)}\left(1+\mathrm{E}_{22}\right)^{-1},
$$

where $\mathrm{E}_{11}, \mathrm{E}_{12}$ and $\mathrm{E}_{22}$ are taken from

$$
\sigma^{2}\left[\begin{array}{ll}
\mathrm{E}_{11} & \mathrm{E}_{12} \\
\mathrm{E}_{12} & \mathrm{E}_{22}
\end{array}\right]:=\left[\begin{array}{cc}
\operatorname{Var}\left(\widehat{\mu}_{G M}^{(k)}\right) & \operatorname{Cov}\left(\widehat{\mu}_{G M}^{(k)}, \widehat{\sigma}_{G M}^{(k)}\right) \\
\operatorname{Cov}\left(\widehat{\mu}_{G M}^{(k)}, \widehat{\sigma}_{G M}^{(k)}\right) & \operatorname{Var}\left(\widehat{\sigma}_{G M}^{(k)}\right)
\end{array}\right],
$$

after using (21), i.e.

$$
\mathrm{E}_{11}=\beta^{2} \frac{T_{0}}{D_{0}}, \quad \mathrm{E}_{12}=\beta \frac{T_{0}-e_{1}}{D_{0}}, \quad \mathrm{E}_{22}=\frac{T_{0}+e_{1}^{2}}{D_{0}} .
$$


Moreover, we have

$$
\operatorname{MSE}\left(\widetilde{\mu}_{B L}^{(k)}\right)=\sigma^{2}\left[\mathrm{E}_{11}-\mathrm{E}_{12}^{2}\left(1+\mathrm{E}_{22}\right)^{-1}\right], \quad \operatorname{MSE}\left(\widetilde{\sigma}_{B L}^{(k)}\right)=\sigma^{2} \mathrm{E}_{22}\left(1+\mathrm{E}_{22}\right)^{-1} .
$$

COROLlaRY 3. When $\alpha$ is known, the best linear invariant estimators for the parameters of the generalized Pareto distribution given by (27) in terms of $k$ th record values have the following form:

$$
\begin{aligned}
& \widetilde{\mu}_{B L}^{(k)}=\widehat{\mu}_{G M}^{(k)}-\widehat{\sigma}_{G M}^{(k)} \frac{1}{\alpha}\left[\frac{T_{0}-\frac{k-2 \alpha}{\alpha}}{T_{0}+D_{0}+\left(\frac{k-2 \alpha}{\alpha}\right)^{2}}\right], \\
& \widetilde{\sigma}_{B L}^{(k)}=\widehat{\sigma}_{G M}^{(k)} \frac{D_{0}}{T_{0}+D_{0}+\left(\frac{k-2 \alpha}{\alpha}\right)^{2}},
\end{aligned}
$$

where

$$
D_{0}=\frac{k-2 \alpha}{\alpha^{2}}\left[k T_{0}-k+2 \alpha\right], \quad T_{0}=\sum_{i=1}^{m}\left(\frac{k-2 \alpha}{k}\right)^{i},
$$

and $\widehat{\sigma}_{G M}^{(k)}$ and $\widehat{\mu}_{G M}^{(k)}$ are the MVLUE for $\sigma$ and $\mu$ given by (28). The mean squared errors of $\widetilde{\mu}_{B L}^{(k)}$ and $\widetilde{\sigma}_{B L}^{(k)}$ are

$$
\begin{aligned}
& \operatorname{MSE}\left(\widetilde{\mu}_{B L}^{(k)}\right)=\sigma^{2} \frac{1}{\alpha^{2}}\left[\frac{T_{0}}{D_{0}}-\frac{\left(T_{0}-\frac{k-2 \alpha}{\alpha}\right)^{2}}{D_{0}\left(D_{0}+T_{0}+\left(\frac{k-2 \alpha}{\alpha}\right)^{2}\right)}\right], \\
& \operatorname{MSE}\left(\widetilde{\sigma}_{B L}^{(k)}\right)=\sigma^{2} \frac{T_{0}+\left(\frac{k-2 \alpha}{\alpha}\right)^{2}}{D_{0}+T_{0}+\left(\frac{k-2 \alpha}{\alpha}\right)^{2}} .
\end{aligned}
$$

REMARK 7. The estimators $\widehat{\mu}_{B L}^{(k)}$ and $\widehat{\sigma}_{B L}^{(k)}$ for $k=1$ were given in [2].

Corollary 4. When $\lambda$ is known, the best linear invariant estimators for the parameters of the Lomax distribution given by (30) in terms of kth record values are

$$
\widetilde{\mu}_{B L}^{(k)}=\widehat{\mu}_{G M}^{(k)}-\widehat{\sigma}_{G M}^{(k)}\left[\frac{T_{0}-e_{1}}{T_{0}+D_{0}+e_{1}^{2}}\right], \quad \widetilde{\sigma}_{B L}^{(k)}=\widehat{\sigma}_{G M}^{(k)} \frac{D_{0}}{T_{0}+D_{0}+e_{1}^{2}} .
$$

The mean squared errors of $\widetilde{\mu}_{B L}^{(k)}$ and $\widetilde{\sigma}_{B L}^{(k)}$ are

$$
\begin{aligned}
& \operatorname{MSE}\left(\widetilde{\mu}_{B L}^{(k)}\right)=\sigma^{2}\left[\frac{T_{0}}{D_{0}}-\frac{\left(T_{0}-e_{1}\right)^{2}}{D_{0}\left(D_{0}+T_{0}+e_{1}^{2}\right)}\right], \\
& \operatorname{MSE}\left(\widetilde{\sigma}_{B L}^{(k)}\right)=\sigma^{2} \frac{T_{0}+e_{1}^{2}}{D_{0}+T_{0}+e_{1}^{2}},
\end{aligned}
$$

where $\widehat{\sigma}_{G M}^{(k)}$ and $\widehat{\mu}_{G M}^{(k)}$ are the MVLUE for $\sigma$ and $\mu$ given by (31) and $D_{0}=$ $e_{1} c_{1} T_{0}-e_{1}^{2}, T_{0}=\sum_{i=1}^{m} e_{i} / c_{i}$ with $e_{1}=(k \lambda-2)^{i}$.

REMARK 8 . The estimators $\widehat{\mu}_{B L}^{(k)}$ and $\widehat{\sigma}_{B L}^{(k)}$ for $k=1$ were presented by Ahsanullah in [2]. 
4.3. Maximum likelihood estimators (MLE). The likelihood function $L$ based on the $k$ th record values for the Burr distribution has the form

$$
\begin{aligned}
L\left(\mu, \sigma \mid \underline{y}^{(k)}\right) & =k^{m}\left(\prod_{i=1}^{m-1} \frac{f\left(y_{i}^{(k)}\right)}{1-F\left(y_{i}^{(k)}\right)}\right)\left[1-F\left(y_{m}^{(k)}\right)\right]^{k-1} f\left(y_{m}^{(k)}\right) \\
& =\frac{k^{m} \lambda^{m}}{\sigma^{m}} \beta^{k \lambda}\left(\beta+\frac{y_{m}^{(k)}-\mu}{\sigma}\right)^{-k \lambda} \prod_{i=1}^{m}\left(\beta+\frac{y_{i}^{(k)}-\mu}{\sigma}\right)^{-1}
\end{aligned}
$$

(see (3)). Hence

$$
\begin{aligned}
\ln L\left(\mu, \sigma, \beta \mid \underline{y}^{(k)}\right)= & \ln k^{m}+\ln \lambda^{m}-\ln \sigma^{m} \\
& -\sum_{i=1}^{m} \ln \left(\beta+\frac{y_{i}^{(k)}-\mu}{\sigma}\right)-k \lambda \ln \left(\beta+\frac{y_{m}^{(k)}-\mu}{\sigma}\right) .
\end{aligned}
$$

Differentiating (33) with respect to $\sigma$ and $\mu$ leads to

$$
\begin{gathered}
\sum_{i=1}^{m}\left(\beta+\frac{y_{i}^{(k)}-\mu}{\sigma}\right)^{-1}+\frac{k \lambda}{\beta+\frac{y_{m}^{(k)}-\mu}{\sigma}}=0, \\
-m \sigma+\sum_{i=1}^{m} \frac{y_{i}^{(k)}-\mu}{\beta+\frac{y_{i}^{(k)}-\mu}{\sigma}}+\frac{k \lambda\left(y_{m}^{(k)}-\mu\right)}{\beta+\frac{y_{m}^{(k)}-\mu}{\sigma}}=0 .
\end{gathered}
$$

When $\lambda$ and $\beta$ are known, the MLE of $\mu$ and $\sigma$ can be obtained by numerical solution of these equations.

\section{References}

[1] M. Ahsanullah, Inference and prediction of the Gumbel distribution based on record values, Pakistan J. Statist. 7 (1991), 53-62.

[2] - Record Statistics, Nova Sci., Commack, NY, 1995.

[3] - Generalized order statistics from exponential distribution, J. Statist. Planning Inference 85 (2000), 85-91.

[4] - The generalized order statistics from two parameter uniform distribution, ibid. 25 (2000), 2311-2318.

[5] M. Ahsanullah and M. Habibullah, Estimators of parameters of Pareto distribution by generalized order statistics, ibid. 29 (2000), 1597-1609.

[6] M. A. M. Ali Mousa, Z. F. Jaheen and A. A. Ahmed, Bayesian estimation, prediction and characterization for the Gumbel model based on records, Statistics 36 (2002), $65-74$.

[7] W. Dziubdziela and B. Kopociński, Limiting properties of the kth record values, Appl. Math. (Warsaw) 15 (1976), 187-190.

[8] E. L. Lloyd, Least-squared estimation of location and scale parameters using order statistics, Biometrika 39 (1952), 88-95. 
[9] I. Malinowska and D. Szynal, On a family of Bayesian estimators and predictors for Gumbel model based on the kth lower records, Appl. Math. (Warsaw) 31 (2004), 107-115.

[10] N. R. Mann, Optimum estimators for linear function of location and scale parameters, Ann. Math. Statist. 40 (1969), 2149-2155.

[11] P. Pawlas and D. Szynal, Relations for single and product moments of kth record values from exponential and Gumbel distributions, J. Appl. Statist. Sci. 7 (1998), $53-62$.

[12] -, - Relations for single and product moments of $k$ th record values from Pareto, generalized Pareto and Burr distributions, ibid. 10 (1999), 53-62.

[13] N. Ravishaner and D. K. Dey, A First Course in Linear Model Theory, Chapman and Hall/CRC, 2002.

Iwona Malinowska

Department of Mathematics

Technical University of Lublin

Nadbystrzycka 38a

20-618 Lublin, Poland

E-mail: i.malinowska@pollub.pl
Piotr Pawlas and Dominik Szynal Department of Mathematics

Maria Curie-Skłodowska University

Pl. M. Curie-Skłodowskiej 1 20-031 Lublin, Poland

E-mail: ppawlas@golem.umcs.lublin.pl szynal@golem.umcs.lublin.pl

Received on 28.1.2005;

revised version on 16.9.2005 